\title{
Fluid Intake Restriction Concomitant to Sweetened Beverages Hydration Induce Kidney Damage
}

\author{
Fernando E. García-Arroyo $\mathbb{D}^{1}{ }^{1}$ Edilia Tapia, ${ }^{1}$ Itzel Muñoz-Jiménez, ${ }^{1}$ \\ Guillermo Gonzaga-Sánchez, ${ }^{1}$ Abraham S. Arellano-Buendía, ${ }^{1}$ Horacio Osorio-Alonso $\mathbb{D},{ }^{1}$ \\ Lino Manterola-Romero, ${ }^{1}$ Carlos A. Roncal-Jiménez, ${ }^{2}$ Richard J. Johnson, ${ }^{2}$ \\ and Laura G. Sánchez-Lozada ${ }^{1}{ }^{1}$
}

${ }^{1}$ Dept. of Cardio-Renal Physiopathology, INC Ignacio Chávez, Mexico City, Mexico

${ }^{2}$ Renal Diseases and Hypertension University of Colorado, Aurora, CO 80045, USA

Correspondence should be addressed to Laura G. Sánchez-Lozada; lgsanchezlozada@gmail.com

Received 7 September 2020; Revised 30 October 2020; Accepted 26 November 2020; Published 7 December 2020

Academic Editor: Antonello Lorenzini

Copyright (c) 2020 Fernando E. García-Arroyo et al. This is an open access article distributed under the Creative Commons Attribution License, which permits unrestricted use, distribution, and reproduction in any medium, provided the original work is properly cited.

\begin{abstract}
Currently, there is the paradox of low water intake but increased intake of sugar-sweetened beverages (SB) in several populations; those habits are associated with an increased prevalence of metabolic derangements and greater chronic disease mortality. Persistent heat dehydration and increased SB intake stimulate the continued release of vasopressin and overactivation of the polyol-fructokinase pathway, synergizing each other, an effect partially mediated by oxidative stress. The objective of the present study was to evaluate whether water restriction concurrent with SB hydration can cause renal damage by stimulating similar pathways as heat dehydration. Three groups of male Wistar rats $(n=6)$ were fluid restricted; from 10 am to $12 \mathrm{pm}$ animals could rehydrate with tap water (W), or sweetened beverages, one prepared with $11 \%$ of a fructose-glucose combination (SB), or with the noncaloric edulcorant stevia (ST). A normal control group of healthy rats was also studied. The animals were followed for 4 weeks. Markers of dehydration and renal damage were evaluated at the end of the study. Fluid restriction and water hydration mildly increased urine osmolality and induced a $15 \%$ fall in $\mathrm{CrCl}$ while increased the markers of tubular damage by NAG and KIM-1. Such changes were in association with a mild overexpression of V1a and V2 renal receptors, polyol fructokinase pathway overactivation, and increased renal oxidative stress with reduced expression of antioxidant enzymes. Hydration with SB significantly amplified those alterations, while in stevia hydrated rats, the changes were similar to the ones observed in water hydrated rats. These data suggest that current habits of hydration could be a risk factor in developing kidney damage.
\end{abstract}

\section{Introduction}

Heat stress and dehydration are accepted risk factors for developing acute kidney injury episodes that may evolve into chronic kidney disease (CKD) $[1,2]$. Dehydration is a condition that increases the risk of death in adults hospitalized for respiratory illness, gastrointestinal, and cardiovascular diseases, as well as diabetes and metabolic disorders [3]. In addition, the condition defined as underhydration (low water intake or serum sodium $>145 \mathrm{mmol} / \mathrm{L}$, spot urine volume $<50 \mathrm{~mL}$, and/or spot urine osmolality $\geq 500 \mathrm{mmol} / \mathrm{kg}$ ), in which total body water is maintained in normal range [4], has also been significantly associated with an increased prevalence of metabolic derangements [4]; this condition was also associated with 4.2 times greater chronic disease mortality [5]. Such data is relevant in the view that less than $50 \%$ of the women and $60 \%$ of the men do not comply with the European Food Safety Agency (EFSA) recommendations on adequate intake of water from fluids [6].

On the other hand, the current consumption of sugarsweetened beverages (SSBs) and fruit juices have significantly increased all over the world [7]. Notably, such food items are 
TABLE 1: Body weight and mean food, fluid, and caloric intake after 30 days of follow-up. Results are presented as mean \pm SD and analyzed by one-way ANOVA followed by Tukey's multiple comparison test. Statistical significance was established as ${ }^{\mathbf{a}} p \leq 0.05 \mathrm{vs}$. C, ${ }^{\mathbf{b}} p \leq 0.01 \mathrm{vs}$. W, and $c_{p} \leq 0.001$ vs. SB.

\begin{tabular}{|c|c|c|c|c|}
\hline Group/parameter & Control & Water & Sweetened beverage & Stevia \\
\hline Body weight (g) & $229.6 \pm 4.7$ & $239.2 \pm 4.5$ & $233.8 \pm 3.5$ & $233 \pm 4.1$ \\
\hline Food intake in $24 \mathrm{~h}(\mathrm{~g})$ & $24.1 \pm 4$ & $23.1 \pm 2$ & $19.7 \pm 2^{\mathbf{a}, \mathbf{b}}$ & $22.8 \pm 2^{\mathfrak{c}}$ \\
\hline Food calories (kcal/day) & $80.2 \pm 3.2$ & $76.3 \pm 6.8$ & $65.1 \pm 5.7^{\mathbf{a}, \mathbf{b}}$ & $75.4 \pm 6.8^{\mathrm{c}}$ \\
\hline Beverage intake in $2 \mathrm{~h}$ rehydration $(\mathrm{mL})$ & - & $8 \pm 1$ & $16 \pm 2^{\mathbf{b}}$ & $16 \pm 2^{\mathbf{b}}$ \\
\hline Beverages calories in $2 \mathrm{~h}$ rehydration $(\mathrm{mL})$ & - & - & $13.5 \pm 5.4$ & - \\
\hline Total fluid intake $(\mathrm{mL})$ & $29 \pm 0.3$ & $23 \pm 1.6^{\mathbf{a}}$ & $31 \pm 2.8^{\mathbf{b}}$ & $31 \pm 2.4^{\mathbf{b}}$ \\
\hline Total calories intake (kcal/day) & $80.2 \pm 3.2$ & $76.3 \pm 6.8$ & $78.6 \pm 5.5$ & $75.4 \pm 6.8$ \\
\hline
\end{tabular}

an essential part of the western type diet [6]. Strikingly, in some countries of Latin America, over $75 \%$ of children drink at least one serving $(250 \mathrm{~mL} /$ day $)$ of SSBs per day, and in Mexico, $82 \%$ of adults consume more than one serving of SSBs daily [6-8]. Thus, currently, there is the paradox of low water intake but increased intake of SSBs in several populations. Significantly, the rise in SSBs intake has been related to cardiovascular disease, metabolic syndrome, cancer, hypertension, and renal damage, including kidney stones as well as all causes of death [9-12].

There is evidence that both chronic heat dehydration and increased SSBs intake stimulate similar mechanisms for the body to maintain its internal milieu. Thus, both insults induce the persistent release of vasopressin (measured as copeptin) and continue overactivation of the polyolfructokinase pathway [13-15]. The chronic activation of both mechanisms causes the development of CKD [1, 2]. Moreover, both mechanisms synergize, resulting in the aggravation of kidney damage [16]. The interlink between both pathways was evidenced when the inhibition of vasopressin receptors with conivaptan blocked the renal overexpression of the polyol-fructokinase pathway enzymes that were in concurrence with the prevention of increased oxidative stress induced by heat dehydration plus SSB hydration [14].

Later on, it was demonstrated that oxidative stress might act as the link between vasopressin and polyol-fructokinase pathways as the supplementation with $\mathrm{N}$-acetylcysteine, in combination with ascorbic acid, resulted in the prevention of renal oxidative stress and the blockade of the renal overexpression of aldose reductase, sorbitol dehydrogenase, and fructokinase, as well as with the preservation of renal function [13]. Such beneficial effects were partially mediated by the nuclear factor erythroid 2-related factor (Nrf2) nuclear translocation. Nrf2 is the master antioxidant regulator and acts by inducing the expression of antioxidant enzymes [17] when it is allowed to translocate into the cell nucleus.

Thus, we hypothesized that dehydration caused by chronic water restriction might act similar to recurrent heat-induced dehydration synergizing with SB's rehydration by stimulating similar pathways causing renal damage.

Mild chronic heat exposure per se can increase oxidative stress [18]. Therefore, the objective of the present study was to evaluate whether mild chronic dehydration induced by water restriction concurrent with sweetened beverage hydra- tion can cause renal damage by stimulating vasopressin and polyol-fructokinase pathways, and by increasing renal oxidative stress.

\section{Materials and Methods}

2.1. Ethical Approval. The studies were conducted in adhesion with the current "Guide of Care and Use of Laboratory Animals" published by the National Institutes of Health and the Mexican Federal Regulation for the animal experimentation and care (NOM-062- ZOO-2001) and the disposal of biological residues (NOM-087-ECOL-1995) and were approved by the Instituto Nacional de Cardiología Ignacio Chavez.

2.2. Experimental Protocol. We included 3 groups of male Wistar rats ( $n=6$ each) that received $15 \mathrm{~mL}$ of tap water for $22 \mathrm{~h} /$ day (approximately one-third of rats regular drinking in $24 \mathrm{~h}$ ). From $10 \mathrm{am}$ and for two hours, animals could replenish with tap water (W), or sweetened beverages, one prepared with $11 \%$ of a fructose-glucose combination (SB), or with the noncaloric edulcorant stevia (ST). As rats tend to increase their fluid intake when sweetened beverages are offered, we included the stevia group for controlling the fluid volume intake. A normal control group of healthy rats was also studied $(n=5)$. The animals were followed for 4 weeks. The volume ingested during rehydration period and the solid food consumed were recorded daily. Body weight was recorded weekly.

2.3. Measurements. After 30 days of follow-up, rats were placed in metabolic cages (Tecniplast. Varese, Italy) by 18 hours to collect urine samples. Systolic blood pressure was measured in conscious rats with a noninvasive tail cuff and validated method [15].

Next, rats were anesthetized with isoflurane and euthanized by exsanguination of the abdominal aorta. The kidneys were washed out by perfusion with cold PBS1x, snap-frozen, and stored in liquid nitrogen until further analysis. The blood samples were centrifuged at $3500 \mathrm{rpm}$ for 5 minutes, and plasma was separated and frozen at $-20^{\circ} \mathrm{C}$ until further processing.

2.4. Markers of Dehydration. Plasma copeptin (a surrogate of vasopressin) was measured with an ELISA kit (Peninsula 

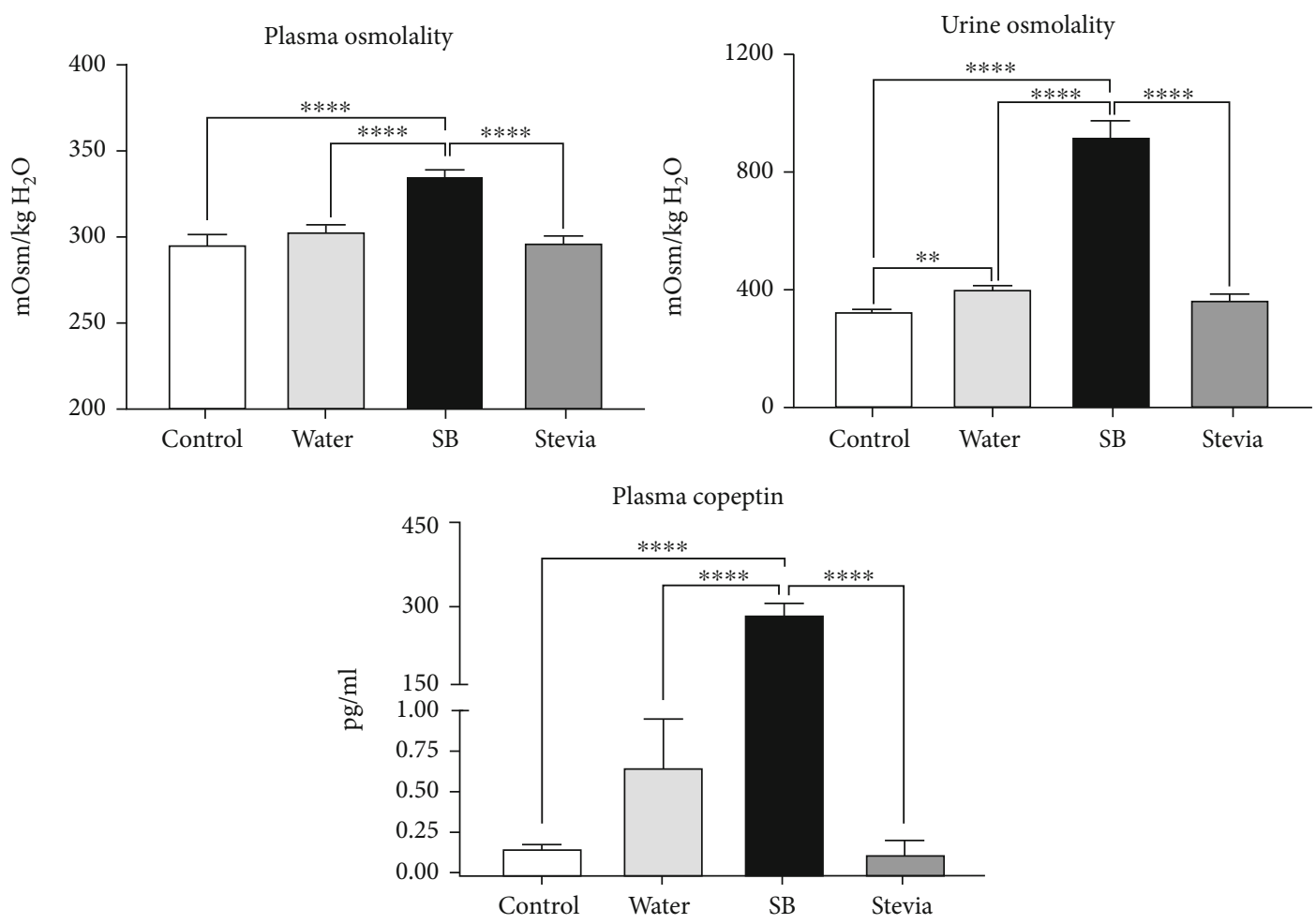

(a)
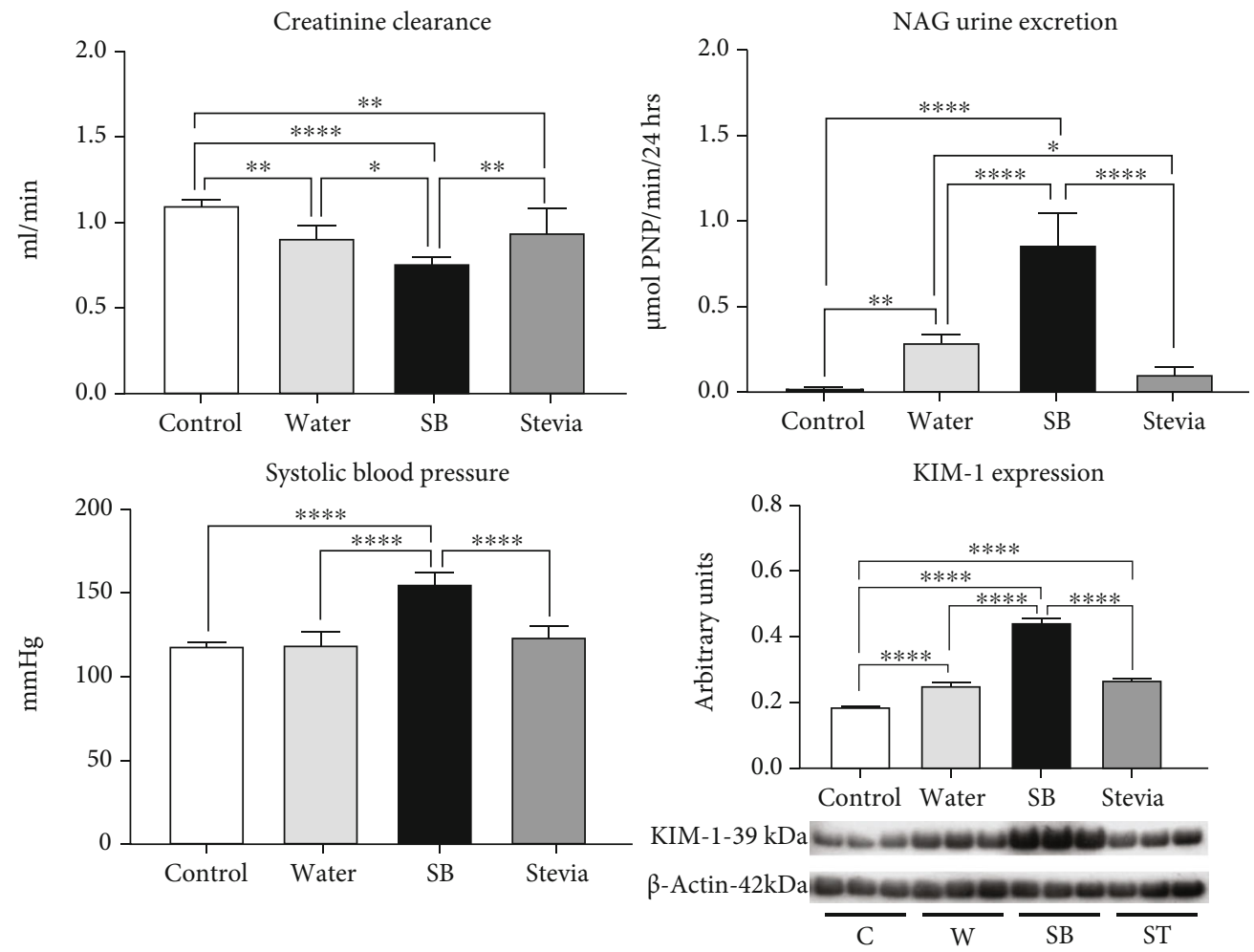

(b)

FIGURE 1: Effects of water restriction and rehydration with a SB on hydration and renal function markers. (a) Markers of hydration: plasma and urine osmolality and plasma copeptin. (b) Markers of renal damage (creatinine clearance, NAG urine excretion, and KIM-1 renal cortex expression) and systolic blood pressure. For western blotting, 3 randomly selected samples per group were analyzed. Results are presented as mean \pm SD and analyzed by one-way ANOVA followed by Tukey's multiple comparison test. Statistical significance was established as ${ }^{*} p \leq 0.05,{ }^{* *} p \leq 0.01,{ }^{* * *} p \leq 0.001$, and ${ }^{* * *} p \leq 0.0001$. 

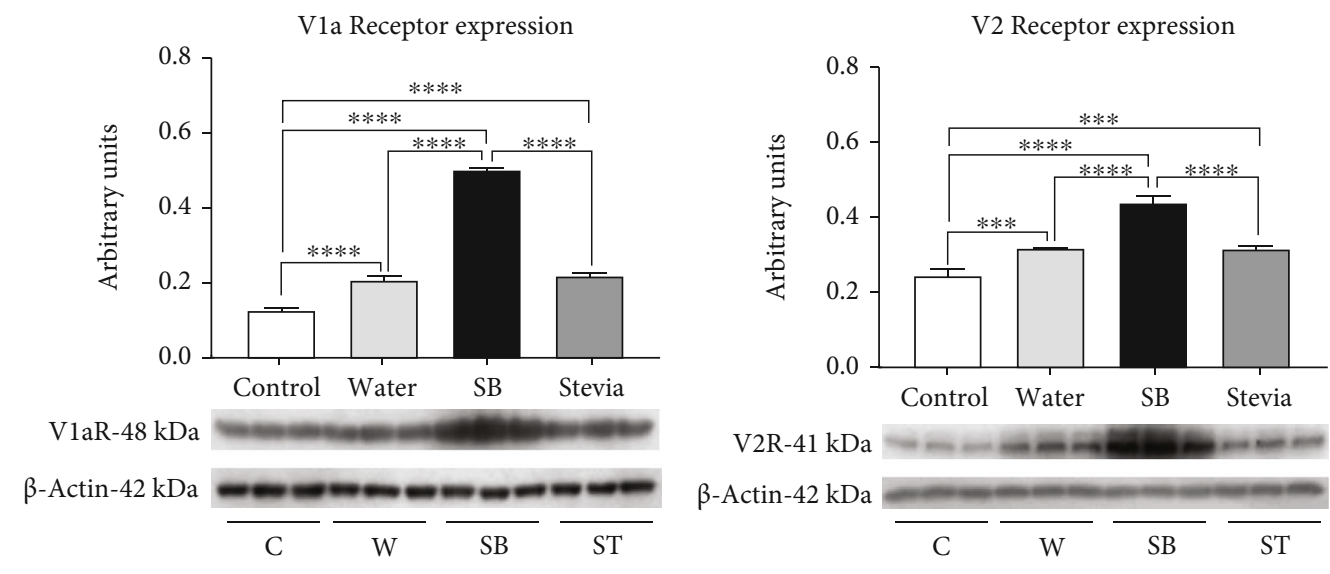

(a)
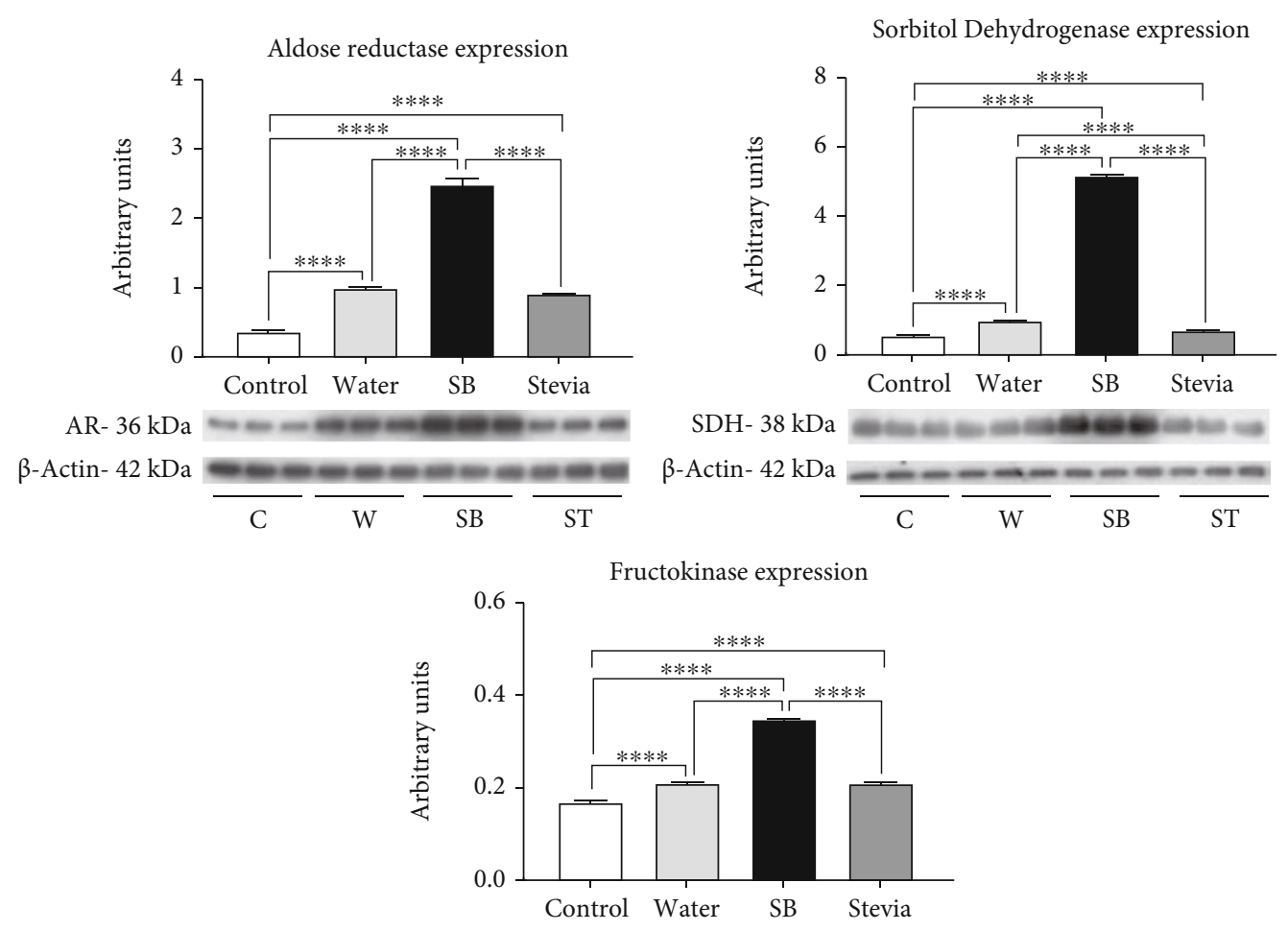

KHK- $33 \mathrm{kDa}=---\infty-\infty-\infty$

$\beta$-Actin- $42 \mathrm{kDa}$

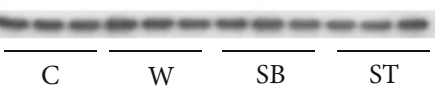

(b)

Figure 2: Continued. 


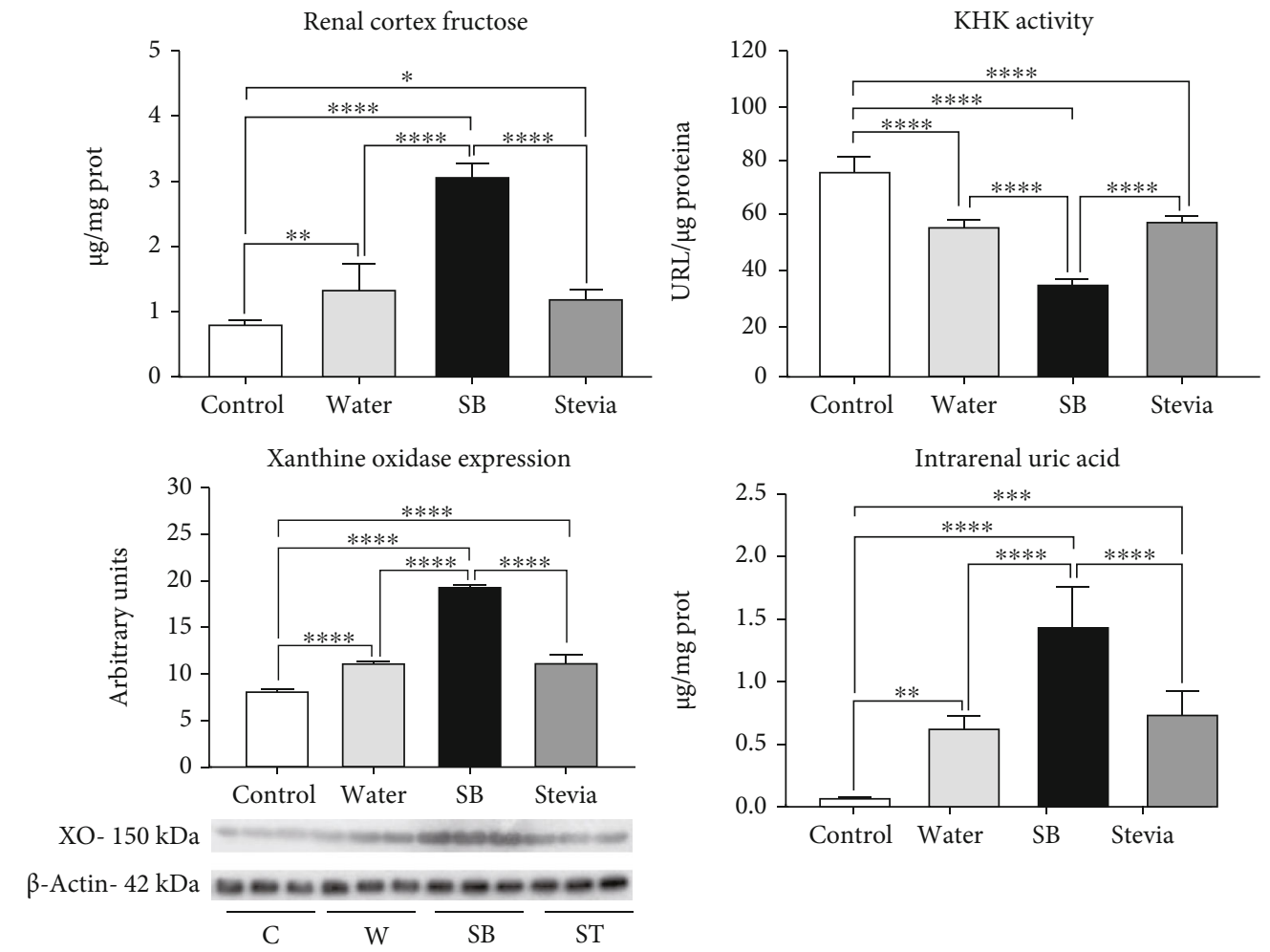

(c)

FIGURE 2: Renal expression of renal vasopressin receptors and polyol-fructokinase pathway enzymes. (a) V1a and V2 vasopressin receptors expression in renal cortex. (b) Aldose reductase, sorbitol dehydrogenase, and fructokinase (KHK) expression in renal cortex. (c) Renal cortex fructose and uric acid concentrations, KHK activity, and xanthine oxidase expression. For western blotting, 3 randomly selected samples per group were analyzed. Results are presented as mean \pm SD and analyzed by one-way ANOVA followed by Tukey's multiple comparison test. Statistical significance was established as ${ }^{*} p \leq 0.05,{ }^{* *} p \leq 0.01,{ }^{* * *} p \leq 0.001$, and ${ }^{* * * *} p \leq 0.0001$.

Laboratories, CA, USA) in samples previously extracted with Sep-pack cartridges C18 accordingly to manufacturer instructions (Water, Milford MA). Plasma and urine sodium concentration was determined by flame photometry. Plasma and urine osmolality were measured in an automatic freezing point osmometer (Löser Osmometer, Berlin, Germany).

2.5. Markers of Renal Function and Renal Tubular Damage. Plasma and urine creatinine were measured using enzymatic kits (SpinReact. Girona, Spain). With creatinine measurements, creatinine clearance $(\mathrm{CrCl})$ was calculated as a proxy of the glomerular filtration rate. Urinary N-Acetyl$\beta$-D-glucosaminidase (NAG) excretion was measured using 4 -nitrophenyl- $\mathrm{N}$-acetyl- $\beta$-D-glucosaminide as a substrate. The data were corrected by urinary volume as previously described [14].

2.6. Western Blotting Analysis. Three samples per group were randomly selected for western blot analysis. Equal amounts of protein $(30 \mu \mathrm{g} / \mathrm{mL})$ were loaded in SDS polyacrylamide gel electrophoresis at 10\% (Mini Protean II, Bio-Rad, Hercules, CA, USA). Proteins were transferred to a nitrocellulose membrane (Criterion Blotter, Bio-Rad, Hercules, CA, USA); membranes were stained with Ponceau red to confirm the transfer and blocked with nonfat dry milk (Bio-Rad, Hercules, CA, USA) $5 \%$ in TBS-tween buffer for 1 hour.
Antibodies were incubated overnight at $4^{\circ} \mathrm{C}$ with $\mathrm{Vla}$ vasopressin receptor (Abcam, ab187753, 1:3000 dilution), V2 vasopressin receptor (Abcam, ab109326, 1:2500 dilution), Aldose reductase (Genetex, GTX113381, 1:2000 dilution), Sorbitol dehydrogenase (Genetex, GTX83588, 1:4000 dilution), Fructokinase (Genetex, GTX109591, 1:5000 dilution), Superoxide Dismutase (Santacruz Biotechnology, sc101523, 1:1000 dilution), Catalase (Santacruz Biotechnology, sc271358, 1:1500 dilution), Glutathione peroxidase (Santacruz Biotechnology, sc22145, 1:1000 dilution), Nox-4 (Novus Biologicals, NB-110-58851, 1:1000 dilution), Bcl2 (Santacruz Biotechnology, sc509, 1:3000 dilution), Bax (Santacruz Biotechnology, sc4239, 1:2000 dilution), Nrf2 (Genetex, GTX103322, 1:2000 dilution), and Kidney Injury Molecule (KIM-1) (Genetex, GTX85067, $1: 3000$ dilution).

To obtain nuclear extracts, renal cortex was homogenized in ice-cold buffer (10 mM HEPES, $0.2 \%$ Triton X-100, $50 \mathrm{mM}$ $\mathrm{NaCl}, 0.5 \mathrm{mM}$ sucrose, $0.1 \mathrm{mM}$ EDTA, and protease and phosphatase inhibitors). Homogenates were centrifuged at $10,000 \mathrm{rpm}$ for $10 \mathrm{~min}$. The pellet was resuspended in ice-cold buffer and incubated for $15 \mathrm{~min}$ and centrifuged for $10 \mathrm{~min}$ at $14,000 \mathrm{rpm}$. The pellet was used for Nrf2 immunoblotting.

2.7. Intrarenal Fructose and Uric Acid. Fructose was extracted with perchloric acid and measured with the anthrone method 

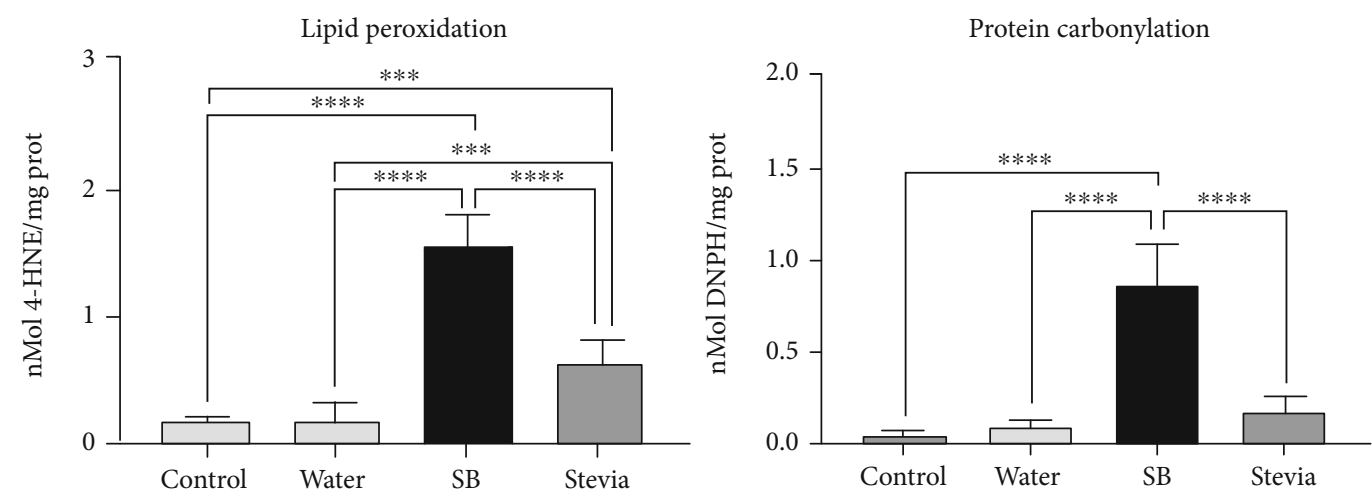

(a)
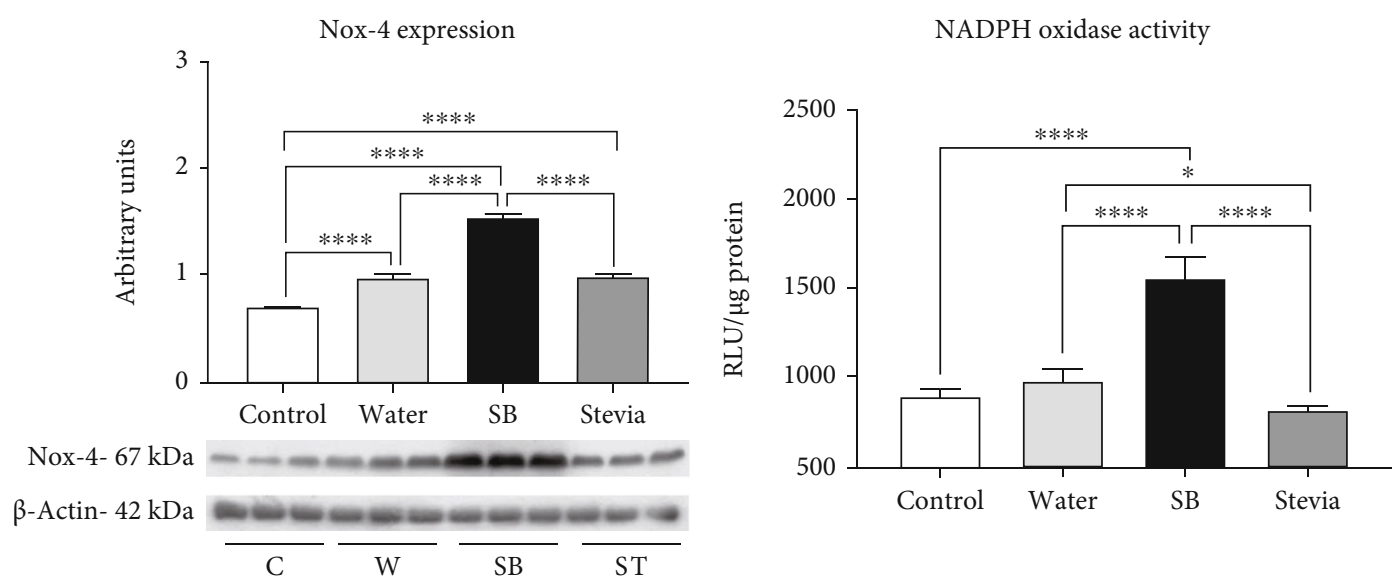

(b)

Figure 3: Oxidative stress in renal cortex. (a) Renal cortex lipid peroxidation measured as 4-hydroxynonenal adducts and protein carbonylation. (b) Renal cortex Nox-4 expression and NADPH oxidase activity. For western blotting, 3 randomly selected samples per group were analyzed. Results are presented as mean \pm SD and analyzed by one-way ANOVA followed by Tukey's multiple comparison test. Statistical significance was established as ${ }^{*} p \leq 0.05,{ }^{* *} p \leq 0.01,{ }^{* * *} p \leq 0.001$, and ${ }^{* * * *} p \leq 0.0001$.

previously described [19]. Uric acid was measured as a byproduct of fructose metabolism; it was extracted by repetitive heat-cold shock [20] from the renal cortex and was measured with a colorimetric kit (Sekisui Diagnostics, Burlington, USA). Fructose and uric acid renal contents were corrected by $\mathrm{mg}$ of protein measured using the method developed by Bradford.

2.8. Markers of Oxidative Stress. Lipid peroxidation and protein carbonylation were measured as irreversible products of oxidative stress in renal cortex homogenates with a colorimetric method previously published $[21,22]$. NADHP oxidase activity was measured in renal cortex homogenates using methods previously described [23].

2.9. Lactate dehydrogenase Activity. Plasma lactate dehydrogenase was measured by an enzymatic kit (Cayman Chemical, Ann Arbor, Michigan, USA) according to manufacturer instructions.

2.10. Fructokinase Activity. Fructokinase activity was measured in renal cortex homogenates by a method previously reported [24].
2.11. Statistical Analysis. Results are presented as mean \pm SD and analyzed by one-way ANOVA followed by Tukey's multiple comparison test. Statistical significance was established as ${ }^{*} p \leq 0.05,{ }^{* *} p \leq 0.01,{ }^{* * *} p \leq 0.001$, and ${ }^{* * * *} p \leq 0.0001$.

\section{Results}

3.1. Water, Food Consumption, and Body Weight. Rats that received tap water ingested $8 \pm 1 \mathrm{~mL}$ (mean) during the rehydration period (two hours each day). The groups that received sweetened waters, either as stevia or $\mathrm{SB}$, consumed similar mean amounts of water for rehydration (16 \pm 2 and $16 \pm 2 \mathrm{~mL}$, respectively), which represented $50 \%$ more volume, compared to tap water group (Table 1). All rats consumed the $15 \mathrm{~mL}$ of water left during the $22 \mathrm{~h}$ of fluid restriction. On the other hand, animals rehydrated with SB water consumed less solid food compared to stevia and water; this trend was found to be significant (Table 1). Nevertheless, total daily caloric intake, provided by solid food plus fluids, was comparable among the four groups (control 80.2 $\pm 3,2$ $\mathrm{kcal} /$ day; water $76.3 \pm 9.61 \mathrm{kcal} /$ day, SB: $78.6 \pm 5.51 \mathrm{kcal} /-$ day and Stevia $75.4 \pm 6.75 \mathrm{kcal} /$ day), and weight gain was not different among the groups (Table 1). 

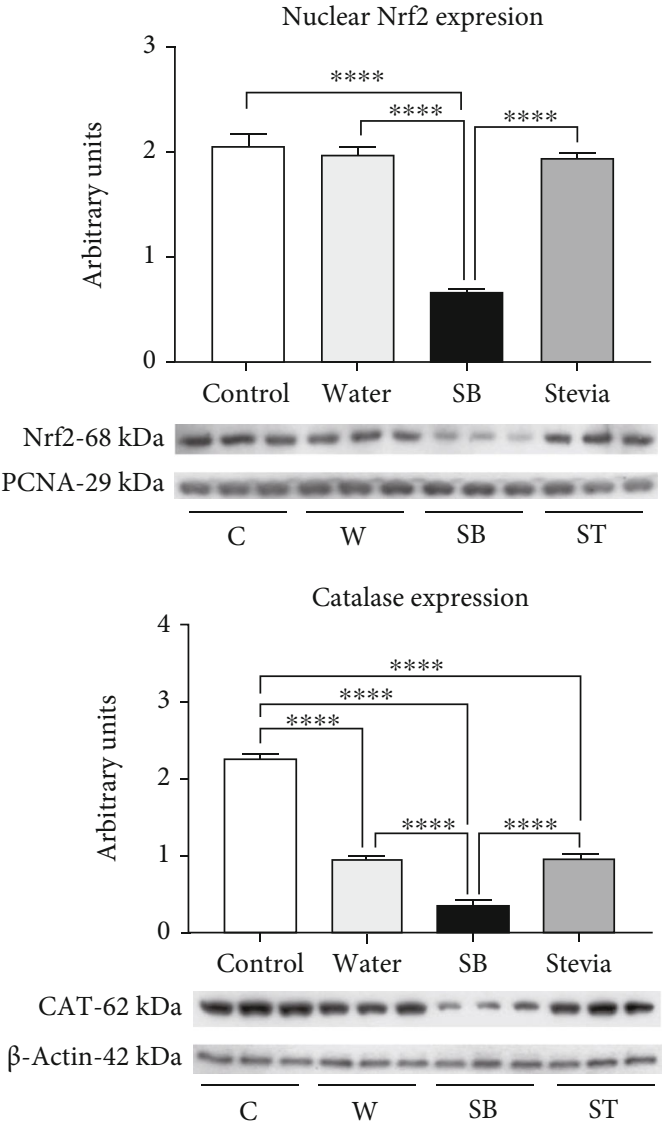
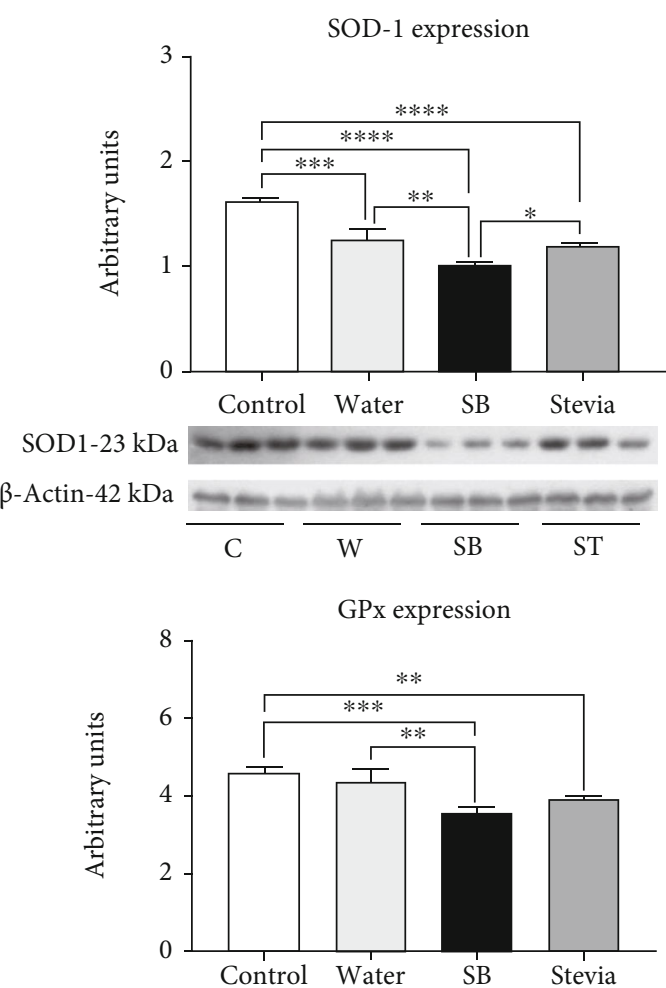

$\mathrm{GPx}-23 \mathrm{kDa}$

$\beta$-Actin- $42 \mathrm{kDa}$

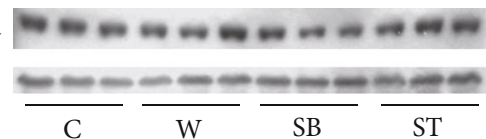

FIgURE 4: Antioxidant enzymes renal expression and Nrf2 nuclear translocation. Renal expression of superoxide dismutase, catalase, and glutathione peroxidase. Nuclear translocation of Nrf2 on renal cortex homogenates. For western blotting, 3 randomly selected samples per group were analyzed. Results are presented as mean \pm SD and analyzed by one-way ANOVA followed by Tukey's multiple comparison test. Statistical significance was established as ${ }^{*} p \leq 0.05,{ }^{* *} p \leq 0.01,{ }^{* * *} p \leq 0.001$, and ${ }^{* * * *} p \leq 0.0001$.

\subsection{Fluid Restriction and Hydration with SB-Induced} Mild Dehydration, Renal Functional Alterations, Mild Hypertension, and Induced Tubular Damage. After 22 hours of fluid restriction and hydration with tap water by 2 hours, there was a mild but significant increment in urine osmolality and an increase of 4 times in plasma copeptin in comparison to control rats. On the other hand, hydration with SB in fluid-restricted rats induced a further increment in plasma and urinary osmolality and plasma copeptin concentration compared vs. C, W, and ST groups (Figure 1(a)). Fluid restriction plus water hydration also induced mild renal alterations as $\mathrm{CrCl}$ fell by $15 \%$ and the markers of tubular damage NAG and KIM-1 mildly but significantly increased. On the other hand, rehydration with SB further reduced creatinine clearance and further increased NAG and KIM-1 (Figure 1(b)). In addition, fluid restriction plus hydration with SB increased systolic blood pressure (SBP) (Figure 1(b)). Fluid-restricted rats hydrated with stevia behaved similarly to $\mathrm{W}$ group at all these parameters.

3.3. Vasopressin and Polyol-Fructokinase Pathways Were Overactivated by Fluid Restriction and Hydration with SB. Vasopressin and polyol-fructokinase pathways with the concomitant synthesis of endogenous fructose and uric acid are mechanisms activated by heat stress-induced dehydra- tion. In the present study, we observed that dehydration induced by water restriction also activated such mechanisms (Figures 2(a)-2(b)). Fluid-restricted rats hydrated with water had a mild but significantly higher expression of renal cortex V1a and V2 vasopressin receptors, aldose reductase, sorbitol dehydrogenase, fructokinase, and as well as a higher concentration of renal fructose (Figures 2(a)2(b)). As fructokinase plays a key role in the renal damage induced by dehydration [24], we indirectly evaluated its activity. In fluid-restricted rats hydrated with water, there was a mild increment in KHK activity (lower ATP tissue concentration). In addition, XO expression, as well as the by-product of fructose metabolism, UA, was also increased in this group (Figure 2(c)).

Previously it was shown that in heat-dehydrated rats, such mechanisms were further overactivated when rats were hydrated with SB $[25,26]$. In the present study, we found a similar effect in fluid-restricted rats. Therefore, we found an additional overexpression in vasopressin receptors and polyol-fructokinase enzymes and fructose renal cortex concentrations. Furthermore, KHK activity was further increased as well as the expression of $\mathrm{XO}$ and $\mathrm{UA}$ renal cortex concentrations. On the other hand, fluidrestricted rats hydrated with stevia showed a similar effect as water hydrated rats (Figure 2(c)). 

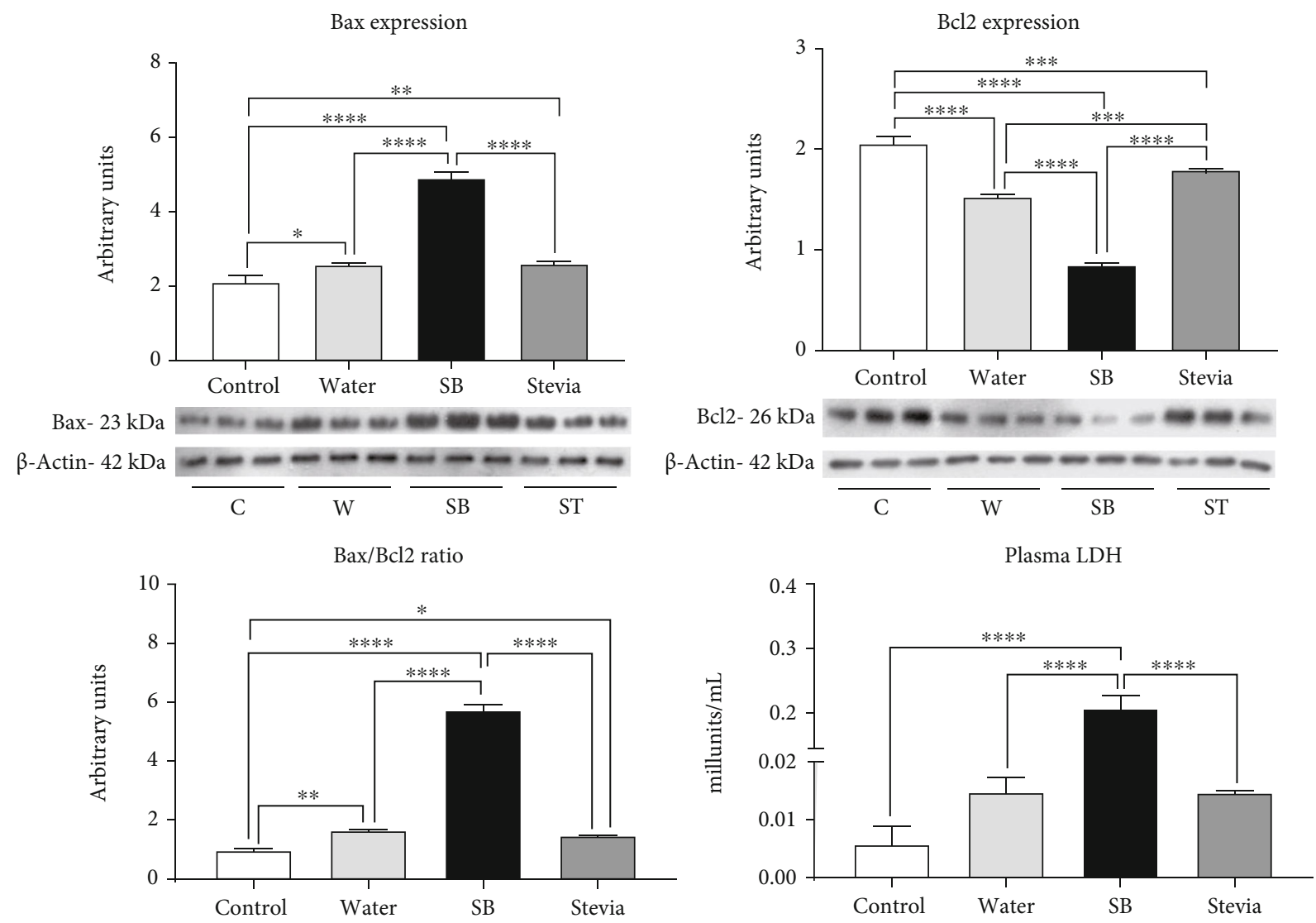

Figure 5: Apoptosis markers. Renal cortex Bax and Bcl2 expressions and Bax/Bcl2 ratio. Plasma LDH concentrations. For western blotting, 3 randomly selected samples per group were analyzed. The results are presented as mean \pm SD and analyzed by one-way ANOVA followed by Tukey's multiple comparison test. Statistical significance was established as ${ }^{*} p \leq 0.05,{ }^{* *} p \leq 0.01,{ }^{* * *} p \leq 0.001$, and ${ }^{* * *} p \leq 0.0001$.

\subsection{Renal Oxidative Stress and Apoptosis Were Increased} by Fluid Restriction and Rehydration with Sweetened Beverages. Lipid peroxidation and protein carbonylation were measured in the renal cortex as markers of oxidative stress. Fluid restriction and hydration with water did not induce an increment of these markers of oxidative stress in comparison to the control group (Figure 3(a)). Despite NOX-4 expression was mildly increased in $\mathrm{W}$ in comparison to C, NADPH oxidase activity remained similar between these two groups. (Figure 3(b)). In agreement with such findings, Nrf2 nuclear translocation (the master regulator of the antioxidant response) in $\mathrm{W}$ group was like $\mathrm{C}$ group; however, SOD-1 and catalase expressions were significantly reduced, while GPX remained without changes in W rats. (Figure 4).

On the other hand, fluid restriction plus SB hydration increased oxidative stress markers by 15 times, and this was in concurrence with NOX-4 overexpression and a significant increment of NADPH oxidase activity (Figure 3(b)). In this group, we observed a significantly decreased nuclear translocation of Nrf2 in concert with a significant reduction in the expression of catalase, SOD-1, and GPx (Figure 4). Finally, hydration with stevia in fluid-restricted rats produced mild oxidative stress accompanied by mildly but significantly higher NOX-4 expression but preserved NADPH oxidase activity (Figure 3(b)) and Nrf2 nuclear translocation, despite
SOD-1, catalase, and Gpx expressions were significantly reduced.

Apoptosis is a secondary effect of Nox4 activation and oxidative stress in tubular cells [27]. Fluid restriction plus water hydration induced a mild but significant increase in Bax expression and a significant decrement in Bcl2 expression suggesting renal cortex apoptosis in this group as indicated by the significant increment in $\mathrm{Bax} / \mathrm{Bcl} 2$ ratio; nevertheless, plasma LDH, a general marker of apoptosis, had a nonsignificant increment (Figure 5). On the other hand, fluid restriction followed by SB hydration induced a further overexpression of Bax and a reduction in the expression of $\mathrm{Bcl} 2$ of $50 \%$ and increased by three times $\mathrm{Bax} / \mathrm{Bcl} 2$ ratio in comparison to $\mathrm{W}$ group. Accordingly, plasma $\mathrm{LDH}$ was twice greater in comparison to W group. Stevia hydrated group showed a similar behavior as $\mathrm{W}$ hydrated group (Figure 5).

\section{Discussion}

In the present work, we demonstrated that intermittent fluid restriction caused mild renal damage prompted by the mechanisms that were previously reported in heat-dehydrated rats. Thus, fluid restriction upregulated the vasopressin renal receptors, overexpressed the renal polyol-fructokinase pathway enzymes, and increased intrarenal oxidative stress. We 
also showed that in fluid restriction, SB hydration also exacerbates such mechanisms. Therefore, these data suggest that poor hydration habits might be a risk factor for developing kidney damage.

In our study, water restriction and intake of SB as fluid of rehydration induced a mild dehydration state similar to heat stress models $[15,28]$ with a significant increase in vasopressin secretion (measured as plasma copeptin) as well as a robust stimulation of renal vasopressin receptors and polyol-fructokinase pathway enzymes including increased KHK activity [24, 29]. Interestingly, rats receiving tap water during rehydration drank much less water but still had a lower plasma copeptin concentration as well as a mild upregulation of renal vasopressin receptors and polyolfructokinase enzymes and KHK activity. On the other hand, rats hydrated with stevia, which had a similar fluid intake as the SB group, had similar responses as the rats hydrated with tap water. Therefore, these findings document that it is not simply how much rehydration fluid is administered, but that the type of fluid also matters as it relates to the regulation of vasopressin and polyol-fructokinase pathways.

Kidney alterations were in parallel to the level of upregulation of vasopressin and polyol fructokinase pathways. Therefore, water restriction and rehydration with a sweetened beverage induced a significant fall in $\mathrm{CrCl}$ and the upregulation of tubular damage markers (KIM-1 and NAG). Our data suggest that the potential mediator between these effects is a concurrent increase of intrarenal oxidative stress. In this regard, increased renal oxidative stress has been associated with kidney damage induced by heat stress models $[14,15]$ as well as other models of kidney damage [20]. In the present study, we observed that the level of activation of the polyol-fructokinase pathway was associated with an increased expression of $\mathrm{XO}$ and uric acid concentrations in the renal cortex. Uric acid is a prooxidant molecule that induces kidney damage $[30,31]$ through the activation of NOX-4. In effect, in the present study, we observed that Nox- 4 was upregulated, and NADPH oxidase renal activity increased, effects better observed in the fluid restricted plus hydration with SB group. This prooxidant effect was supported by a significant increase in the tissue markers of lipid peroxidation and protein carbonylation. In previous studies from our group, we showed that heat stress and hydration with fructose-containing beverages induce the overexpression of Nox-4 as well as the catalytic accompanying subunits p22 and Gp-91 [14]. In concert with a prooxidative milieu caused by fluid restriction and SB hydration, we also found a significant reduction of the antioxidant system. Therefore, SOD, CAT, and GPx expressions were significantly decreased in the fluid-restricted group hydrated with SB. Such an effect partially resulted from a decreased nuclear translocation of Nrf2, which is their principal transcription factor. Another detrimental effect of Nox-4-mediated oxidative stress is to promote apoptosis in proximal tubule cells [27], a secondary effect of an imbalance of $\mathrm{Bax} / \mathrm{Bcl}-2$ [27]. In this regard, mitochondrial damage induced by uric acid in renal cells also is associated with an increased apoptosis [32]. In the present study, we found an imbalance of $\mathrm{Bax}$ and $\mathrm{Bcl} 2$ expressions related to more significant oxidative stress in the fluid- restricted plus SB hydrated group, suggesting an increase in renal apoptosis. This finding was further supported by an increase in LDH activity in the plasma samples from the same group.

Some limitations of this study should be stated. This is an experimental study in which the water restriction and the hydration beverages content were variables fully controlled. Therefore, this is somehow different from the situation found in free-living individuals. As such, the findings cannot be immediately extended to humans.

\section{Conclusions}

In summary, the chronic fluid restriction can induce mild renal alterations that are aggravated when a sweetened beverage is used as hydration fluid. The kidney damage was partially mediated by increased vasopressin release, overactivation of the polyol-fructokinase pathway, increased oxidative stress, and apoptosis. These data suggest that current habits of hydration could be a risk factor in developing kidney damage.

\section{Data Availability}

Data is available from the corresponding author upon request.

\section{Disclosure}

The funders had no role in the design of the study; in the collection, analysis, or interpretation of data; in the writing of the manuscript, or in the decision to publish the results.

\section{Conflicts of Interest}

Some authors (L.G.S.L, C.A.R.-J., and R.J.J.) are members of Colorado Research Partners, LLC, a start-up company developing inhibitors of fructose metabolism.

\section{Authors' Contributions}

Conceptualization was performed by Fernando E GarcíaArroyo, Richard J Johnson, and Laura Gabriela SánchezLozada. Data curation was performed by Fernando E García-Arroyo and Laura Gabriela Sánchez-Lozada. Formal analysis was performed by Fernando E García-Arroyo and Laura Gabriela Sánchez-Lozada. Funding acquisition was performed by Laura Gabriela Sánchez-Lozada. Investigation was performed by Fernando E García-Arroyo, Edilia Tapia, Itzel Muñoz-Jimenez, Guillermo Gonzaga-Sánchez, Abraham S Arellano-Buendía, and Lino Manterola-Romero. Methodology was performed by Fernando E García-Arroyo, Edilia Tapia, Guillermo Gonzaga-Sánchez, Abraham S Arellano-Buendía, and Laura Gabriela Sánchez- Lozada. Project administration was performed by Laura Gabriela SánchezLozada. Fernando E García-Arroyo and Laura Gabriela Sánchez-Lozada wrote the original draft. Horacio OsorioAlonso, Carlos A Roncal-Jiménez, Richard J Johnson, and Laura Gabriela Sánchez-Lozada wrote, reviewed, and edited the manuscript. 


\section{Acknowledgments}

This research was funded by Fondos de Gasto Directo autorizado a la Subdirección de Investigación Básica del Instituto Nacional de Cardiología Ignacio Chávez.

\section{References}

[1] J. Glaser, J. Lemery, B. Rajagopalan et al., "Climate change and the emergent epidemic of CKD from heat stress in rural communities: the case for heat stress nephropathy," Clinical Journal of the American Society of Nephrology, vol. 11, no. 8, pp. 1472-1483, 2016.

[2] M. Madero, F. E. García-Arroyo, and L. G. Sánchez-Lozada, "Pathophysiologic insight into MesoAmerican nephropathy," Current Opinion in Nephrology and Hypertension, vol. 26, no. 4, pp. 296-302, 2017.

[3] J. Warren, I. Guelinckx, B. Livingstone et al., "Challenges in the assessment of total fluid intake in children and adolescents: a discussion paper," European Journal of Nutrition, vol. 57, Suppl 3, pp. 43-51, 2018.

[4] S. A. Kavouras, "Hydration, dehydration, underhydration, optimal hydration: are we barking up the wrong tree?," European Journal of Nutrition, vol. 58, no. 2, pp. 471-473, 2019.

[5] J. D. Stookey, S. A. Kavouras, H. Suh, and F. Lang, "Underhydration is associated with obesity, chronic diseases, and death within 3 to 6 years in the U.S. population aged 51-70 years," Nutrients, vol. 12, no. 4, p. 905, 2020.

[6] J. D. Stookey and J. König, "Describing water intake in six countries: results of Liq.In ${ }^{7}$ surveys, 2015-2018," European Journal of Nutrition, vol. 57, no. S3, pp. 35-42, 2018.

[7] G. M. Singh, R. Micha, S. Khatibzadeh et al., "Global, regional, and national consumption of sugar-sweetened beverages, fruit juices, and milk: a systematic assessment of beverage intake in 187 countries," PLoS One, vol. 10, no. 8, article e0124845, 2015.

[8] H. Martinez, C. Morin, J. Gandy et al., "Fluid intake of Latin American adults: results of four 2016 Liq.In7 national crosssectional surveys," European Journal of Nutrition, vol. 57, no. S3, pp. 65-75, 2018.

[9] R. J. Johnson, L. G. Sanchez-Lozada, and T. Nakagawa, “The effect of fructose on renal biology and disease," Journal of the American Society of Nephrology, vol. 21, no. 12, pp. 20362039, 2010.

[10] K. Bratoeva, G. S. Stoyanov, A. Merdzhanova, and M. Radanova, "Manifestations of renal impairment in fructose-induced metabolic syndrome," Cureus, vol. 9, no. 11, p. e1826, 2017.

[11] R. J. Johnson, S. E. Perez-Pozo, J. L. Lillo et al., "Fructose increases risk for kidney stones: potential role in metabolic syndrome and heat stress," BMC Nephrology, vol. 19, no. 1, p. 315, 2018.

[12] G. M. Singh, R. Micha, S. Khatibzadeh, S. Lim, M. Ezzati, and D. Mozaffarian, "Estimated Global, Regional, and National Disease Burdens Related to Sugar-Sweetened Beverage Consumption in 2010," Circulation, vol. 132, no. 8, pp. 639-666, 2015.

[13] F. E. García-Arroyo, G. Gonzaga, I. Muñoz-Jiménez et al., "Antioxidant supplements as a novel mean for blocking recurrent heat stress-induced kidney damage following rehydration with fructose-containing beverages," Free Radical Biology \& Medicine, vol. 141, pp. 182-191, 2019.
[14] F. E. García-Arroyo, E. Tapia, M. G. Blas-Marron et al., "Vasopressin mediates the renal damage induced by limited fructose rehydration in recurrently dehydrated rats," International Journal of Biological Sciences, vol. 13, no. 8, pp. 961-975, 2017.

[15] F. E. García-Arroyo, M. Cristóbal, A. S. Arellano-Buendía et al., "Rehydration with soft drink-like beverages exacerbates dehydration and worsens dehydration-associated renal injury," American Journal of Physiology-Regulatory, Integrative and Comparative Physiology, vol. 311, no. 1, pp. R57R65, 2016.

[16] L. G. Sánchez-Lozada, C. A. Roncal-Jimenez, F. E. GarcíaArroyo, T. Jensen, M. A. Lanaspa, and R. J. Johnson, "The perils of rehydrating with soft drinks following heat and exercise," American Journal of Physiology-Regulatory, Integrative and Comparative Physiology, vol. 316, no. 3, pp. R187-R188, 2019.

[17] J. D. Hayes and A. T. Dinkova-Kostova, "The Nrf2 regulatory network provides an interface between redox and intermediary metabolism," Trends in Biochemical Sciences, vol. 39, no. 4, pp. 199-218, 2014.

[18] Y. Kasanuma, C. Watanabe, C. Y. Kim, K. Yin, and H. Satoh, "Effects of mild chronic heat exposure on the concentrations of thiobarbituric acid reactive substances, glutathione, and selenium, and glutathione peroxidase activity in the mouse liver," The Tohoku Journal of Experimental Medicine, vol. 185, no. 2, pp. 79-87, 1998.

[19] M. N. Halhoul and I. Kleinberg, "Differential determination of glucose and fructose, and glucose- and fructose-yielding substances with anthrone," Analytical Biochemistry, vol. 50, no. 2, pp. 337-343, 1972.

[20] P. Cirillo, M. S. Gersch, W. Mu et al., "Ketohexokinase-dependent metabolism of fructose induces proinflammatory mediators in proximal tubular cells," Journal of the American Society of Nephrology, vol. 20, no. 3, pp. 545-553, 2009.

[21] E. Molina-Jijón, E. Tapia, C. Zazueta et al., "Curcumin prevents $\mathrm{Cr}$ (VI)-induced renal oxidant damage by a mitochondrial pathway," Free Radical Biology \& Medicine, vol. 51, no. 8, pp. 1543-1557, 2011.

[22] Y. Y. Sautin, T. Nakagawa, S. Zharikov, and R. J. Johnson, "Adverse effects of the classic antioxidant uric acid in adipocytes: NADPH oxidase-mediated oxidative/nitrosative stress," American Journal of Physiology. Cell Physiology, vol. 293, no. 2, pp. C584-C596, 2007.

[23] M. R. Abid, K. C. Spokes, S. C. Shih, and W. C. Aird, "NADPH oxidase activity selectively modulates vascular endothelial growth factor signaling pathways," The Journal of Biological Chemistry, vol. 282, no. 48, pp. 35373-35385, 2007.

[24] A. Andres-Hernando, N. Li, C. Cicerchi et al., "Protective role of fructokinase blockade in the pathogenesis of acute kidney injury in mice," Nature Communications, vol. 8, no. 1, 2017.

[25] C. A. Roncal Jimenez, T. Ishimoto, M. A. Lanaspa et al., "Fructokinase activity mediates dehydration-induced renal injury," Kidney International, vol. 86, no. 2, pp. 294-302, 2014.

[26] R. J. Johnson, P. Stenvinkel, T. Jensen et al., "Metabolic and kidney diseases in the setting of climate change, water shortage, and survival factors," Journal of the American Society of Nephrology, vol. 27, no. 8, pp. 2247-2256, 2016.

[27] Z. Li, Y. Sheng, C. Liu et al., "Nox4 has a crucial role in uric acid-induced oxidative stress and apoptosis in renal tubular cells," Molecular Medicine Reports, vol. 13, no. 5, pp. 43434348, 2016. 
[28] T. Milagres, F. E. García-Arroyo, M. A. Lanaspa et al., "Rehydration with fructose worsens dehydration-induced renal damage," BMC Nephrology, vol. 19, no. 1, p. 180, 2018.

[29] R. R. Pryor, J. L. Pryor, L. W. Vandermark et al., "Acute kidney injury biomarker responses to short-term heat acclimation," International Journal of Environmental Research and Public Health, vol. 17, no. 4, p. 1325, 2020.

[30] M. Cristóbal-García, F. E. García-Arroyo, E. Tapia et al., "Renal oxidative stress induced by long-term hyperuricemia alters mitochondrial function and maintains systemic hypertension," Oxidative Medicine and Cellular Longevity, vol. 2015, 8 pages, 2015.

[31] G. Glantzounis, E. Tsimoyiannis, A. Kappas, and D. Galaris, "Uric acid and oxidative stress," Current Pharmaceutical Design, vol. 11, no. 32, pp. 4145-4151, 2005.

[32] L. Yang, B. Chang, Y. Guo, X. Wu, and L. Liu, "The role of oxidative stress-mediated apoptosis in the pathogenesis of uric acid nephropathy," Renal Failure, vol. 41, no. 1, pp. 616-622, 2019. 